CARPATHIAN J. MATH.

Volume 37 (2021), No. 3,

Pages 427 - 440
Online version at https : //www. carpathian. cunbm. utcluj. ro/

Print Edition: ISSN 1584 - 2851; Online Edition: ISSN 1843 - 4401

DOI: https://doi.org/10.37193/CJM.2021.03.06

\title{
A stochastic control problem with regime switching
}

\author{
Dragoş-Pătru Covei and Traian A. Pirvu
}

\begin{abstract}
This paper studies a stochastic control problem with regime switching in a fairly general abstract setting. Such problems may arise from production planning management. We perform a full mathematical analysis of this stochastic control problem via the HJB equation and verification. The connection of the optimal controls and subgame perfect controls is discussed, and it is shown that the optimal controls solve the generalized $\mathrm{HJB}$ equation as well. In a special case we provide a closed form solution.
\end{abstract}

\section{INTRODUCTION}

Stochastic control problems arise in decision making process, and thus they model a myriad of real world problems. We have seen a growth in the last decades of applications of stochastic control in finance, economics, and management science. Our setting is fairly general, but it was inspired by a number of recent works in production planning management. Here is the list of the papers ([1], [2], [4], [6], [7], [10] and [24]) that deal with the cost minimization problem of a factory which tries to find the optimal production rate in a stochastic demand framework, and facing inventory costs as well. These works served as the motivation for this paper, which tries to provide an abstract setting, and the mathematical analysis for the stochastic control, cost optimization problem.

Let us present an overview of stochastic control problems of the type we address. A pioneer paper is [24] which considers controls to be confined in a bounded domain of $\mathbb{R}^{N}$. The results of [24] have been generalized to the case $\mathbb{R}^{N}$ by [1]. This work shows that the value function solves the corresponding Hamilton Jacobi Belmann (HJB) equation, thus providing a partial differential equation (PDE) characterization for the value function. Next we will focus on stochastic control problems with regime switching since this is the paradigm of our paper. Regime switching refers to situations when there are several regimes in the model which make some of the model parameters change with the regime. Such a modelling approach is not new; in finance for instance periods of bull and bear markets can be modelled as the two switching regimes/states of the model. The interested reader can find out more about this financial modelling in [30] and [32] . In production management the regime switching can refer to situations when there is an increase in demand during some economic cycles. In such a context we point to the following papers [3], [5] and [17]. An extension of [24] to multiple regimes was implemented by [3] which solves the inventory problem of a company. The work [34], in a regime switching environment, solves the stochastic control problem faced by an insurance company which tries to minimize the total cost up to a stopping time. Recently, [29] analyzes a stochastic control problem in a multi-dimensional diffusion setting with regime-switching and possible unbounded controls. They use dynamic programming to characterize the value function as a viscosity solution of nonlinear quasi-variational inequalities.

Received: 06.07.2020. In revised form: 22.12.2020. Accepted: 03.06.2021

2010 Mathematics Subject Classification. 35J57, 35J47, 60J70.

Key words and phrases. Stochastic control, HJB equation, generalized HJB equation, partial differential equations, elliptic systems.

Corresponding author: Dragos-Patru Covei; coveidragos@yahoo.com 
In a stochastic setting this paper considers the minimization problem of a cost functional over an infinite time horizon. The cost functional involves a vector controlled state process, and its corresponding control. The uncertainty is driven by a multi dimensional Brownian motion and a Markov chain modelling the regime switching. We tackle this problem via dynamic programming. By employing probabilistic techniques (the martingale/supermartingale principle) we derive the corresponding Hamilton Jacobi Bellman (HJB) equation. The later turns out to be in our context an elliptic semilinear system of partial differential equations. There seems to be no previous mathematical results about the existence of positive solutions for thus semilinear system. This should not surprise us since there are some difficulties in analyzing this class of systems that we point to in the body of the paper. We provide a verification result, meaning that we show that the solution of the HJB equation gives in turn the optimal controls. Some complications due to the infinite planning horizon arise in completing this process, and we deal with them via the well known transversality condition.

In some applications / settings the optimal decisions are time inconsistent, meaning that they will not be implemented, unless there is a commitment mechanism, because they fail the optimality when the optimization criterion is updated. One way of addressing this problem is considering the subgame perfect controls; these are Nash equilibrium controls. The subgame perfect control are time consistent; for more on this see [11], [12] and [30]. The subgame perfect controls are characterized by a generalized HJB, which is a fixed point problem, involving the fixed point of a functional coupled with a Hamiltonian condition and the associated flow. In our setting the optimal controls are time consistent and as such they are also subgame perfect. We show that the optimal controls solve the generalized HJB equation and as such they admit a fixed point characterization.

The contributions of this paper are three folds: 1) we characterize the value function of a infinite horizon stochastic control problem through the HJB equation, through probabilistic methods (martingale/supermartingale principle), and established the verification (i.e. the solution of the HJB equation is the value function); 2) we introduced the subgame perfect controls and have shown that in our setting they coincide with the optimal controls; 3) an example was provided where we managed to solve the stochastic control problem in closed form.

The reminder of the paper is organized as follows. Section 2 describes the model and formulated the stochastic control problem. The next 3 sections provide the analysis of the stochastic control problem. A section is dedicated to the connection of the subgame perfect controls and the optimal controls, as well as the characterization of the later through the generalized HJB equation leading to a fixed point problem. The paper ends with a closed form solution in a special case.

\section{THE MODEL AND PROBLEM FORMULATION}

Let us present the setting. Consider $W$ a $N$-dimensional Brownian motion on a filtered probability space

$$
\left(\Omega,\left\{\mathcal{F}_{t}\right\}_{0 \leq t \leq T}, \mathcal{F}, P\right),
$$

where $\left\{\mathcal{F}_{t}\right\}_{0 \leq t \leq T}$ is a completed filtration, and $T=\infty$ (we deal with the infinite horizon case). We allow for regime switching in our model; regime switching refers to the situation when the characteristics of the state process are affected by several regimes (e.g. in finance bull and bear market with higher volatility in the bear market). The regime switching is captured by a continuous time homogeneous right continuous Markov chain $\epsilon(t)$ adapted 
to $\mathcal{F}_{t}$ with two regimes good and bad, i.e., for every

$$
t \in[0, \infty) \text { and } \epsilon(t) \in\{1,2\} .
$$

In a specific application, $\epsilon(t)=1$ could represent a regime of economic growth while $\epsilon(t)=2$ could represent a regime of economic recession. In another application, $\epsilon(t)=1$ could represent a regime in which consumer demand is high while $\epsilon(t)=2$ could represent a regime in which consumer demand is low.

The Markov chain's rate matrix is

$$
A=\left(\begin{array}{cc}
-a_{1} & a_{1} \\
a_{2} & -a_{2}
\end{array}\right)
$$

for some $a_{1}>0, a_{2}>0$. Diagonal elements $A_{i i}$ are defined such that

$$
A_{i i}=-\sum_{j \neq i} A_{i j}
$$

where

$$
A_{11}=-a_{1}, A_{12}=a_{1}, A_{21}=a_{2}, A_{22}=-a_{2} .
$$

In this case, if $p_{t}=\mathbb{E}[\epsilon(t)] \in \mathbb{R}^{2}$, then

$$
\frac{d p_{t}}{d t}=A \epsilon(t)
$$

Moreover

$$
\epsilon(t)=\epsilon(0)+\int_{0}^{t} A \epsilon(u) d u+M(t),
$$

where $M(t)$ is a martingale with respect to $\mathcal{F}_{t}$.

Let us consider a Markov modulated controlled diffusion with controls in feed-back form

$$
d X^{i}(t)=c_{\epsilon(t)}^{i}(X(t)) d t+k_{\epsilon(t)} d W^{i}(t), i=1, \ldots N,
$$

for some constants $k_{1}>0, k_{2}>0$, and $X(0)=x \in \mathbb{R}^{N}$. Here, at every time $t$, the control $c_{\epsilon(t)}$, is given in feedback form, and the volatility $k_{\epsilon(t)}$ depend on the regime $\epsilon(t)$. We allow the demand to take on negative values. We consider the class of admissible controls, $\mathcal{A}$, the feedback controls for which the SDE (2.6) has a solution. The vector controlled process $X(t)$ with components $X^{i}(t), i=1, \ldots N$, and $t>0$ is called the state process.

Next, for each $c \in \mathcal{A}$, we introduce the cost functional, formally defined by

$$
J(x, c, i)=E\left[\int_{0}^{\infty} e^{-\lambda_{\epsilon(t)} t}\left[f_{\epsilon(t)}(X(t))+\frac{1}{2}|c|_{\epsilon(t)}^{2}(X(t))\right] d t \mid X(0)=x, \epsilon(0)=i\right] .
$$

where the loss $f_{1}, f_{2}: \mathbb{R}^{N} \rightarrow[0, \infty)$ are continuous, convex functions satisfying

$$
\text { there exists } M_{i}>0 \text { such that } f_{i}(x) \leq M_{i}\left(|x|^{2}+1\right), i=1,2 .
$$

and $\lambda_{\epsilon(t)}$ is a regime dependent (taking on two values $\lambda_{1}>0$ and $\lambda_{2}>0$ ), constant psychological rate of time discount, whence the exponential discounting.

Our objective is to minimize the functional $J$, i.e. determine the value function

$$
z_{i}(x)=\inf J(x, c, i),
$$

and to find the optimal control. The infimum is taken over all admissible controls $c \in \mathcal{A}$. Notice that the discount rate depends on the regime; for more on this modelling approach see [30]. 
Remark 2.1. This setting can be specialized to model a production cost minimization problem of a factory. In such a case the control $c$ would be the production rate adjusted for demand (which can be stochastic), and the state process $X$ would be the inventory, i.e., the number of products of certain types produced. We point the reader to [2], [4], [6], [7] and [10] for more on this. Regime switching may be added in the modelling of the management production problem; see for instance [3], [5] and [17] for more on this.

\section{DYNAMIC PROGRAMMING APPROACH AND THE HJB EQUATION}

In order to obtain the HJB equation we apply the martingale/supermartingale principle; search for a function $u(x, i)$ such that the stochastic process $M^{c}(t)$ defined below

$$
M^{c}(t)=e^{-\lambda_{\epsilon(t)} t} u(X(t), \epsilon(t))-\int_{0}^{t} e^{-\lambda_{\epsilon(u)} u}\left[f_{\epsilon(t)}(X(u))+\frac{1}{2}|c|_{\epsilon(u)}^{2}(X(u))\right] d u,
$$

is supermartingale and martingale for the optimal control. If this is achieved together with the following transversality condition

$$
\lim _{t \rightarrow \infty} E\left[e^{-\lambda_{\epsilon(t)} t} u(X(t), \epsilon(t))\right]=0,
$$

and some estimates on the value function yield that

$$
z_{i}(x)=-u(x, i)=\inf _{c \in \mathcal{A}} J(x, c, i) .
$$

The proof of this statement is done in the Verification subsection.

The infinitesimal generator $L$ of diffusion $X$ is second order differential operator defined by

$$
\begin{aligned}
& L^{c} v(x, 1)=\frac{1}{2} k_{1} \Delta v(x, 1)+c_{1} \nabla v(x, 1)+A_{11} v(x, 1)+A_{12} v(x, 2), \\
& L^{c} v(x, 2)=\frac{1}{2} k_{2} \Delta v(x, 2)+c_{2} \nabla v(x, 2)+A_{22} v(x, 2)+A_{21} v(x, 1),
\end{aligned}
$$

(see [26] for more on this). Following this we can state Itô's formula for Markov modulated diffusion

$$
d v(X(t), \epsilon(t))=L^{c} v(X(t), \epsilon(t)) d t+k_{\epsilon(t)} \nabla v(X(t), \epsilon(t)) d W(t) .
$$

The supermartingale/martingale requirement of $M^{c}(t)$ process in (3.10), leads to the following HJB equation

$$
\frac{k_{i}}{2} \Delta u(x, i)+\sup _{c \in \mathcal{A}}\left[\nabla u(x, i) c-\frac{|c|^{2}}{2}\right]=f_{i}(x)+\left(\lambda_{i}+a_{i}\right) u(x, i)-a_{i} u(x, j),
$$

for $i, j \in\{1,2\}$. First order condition yields the candidate optimal control

$$
\hat{c}_{i}(x)=\nabla u(x, i)=-\nabla z_{i}(x),
$$

and this leads to the system

$$
\frac{k_{i}}{2} \Delta u(x, i)+\frac{|\nabla u(x, i)|^{2}}{2}=f_{i}(x)+\left(\lambda_{i}+a_{i}\right) u(x, i)-a_{i} u(x, j),
$$

for $i, j \in\{1,2\}$. Alternatively this system can be written in terms of $z_{i}(x),(i=1,2)$ to get

$$
\left\{\begin{array}{l}
-\frac{k_{1}}{2} \Delta z_{1}(x)+\frac{\left|\nabla z_{1}(x)\right|^{2}}{2}=f_{1}(x)-\left(\lambda_{1}+a_{1}\right) z_{1}(x)+a_{1} z_{2}(x), \\
-\frac{k_{2}}{2} \Delta z_{2}(x)+\frac{\left|\nabla z_{2}(x)\right|^{2}}{2}=f_{2}(x)-\left(\lambda_{2}+a_{2}\right) z_{2}(x)+a_{2} z_{1}(x),
\end{array} \quad x \in \mathbb{R}^{N} .\right.
$$


For the particular case $a_{1}=a_{2}=0$ this system can be solved using the ideas introduced in $[8,9]$, see also the recent paper of [4]. Our mathematical results described in the next section applies to all

$$
k_{1}, k_{2}, a_{1}, a_{2}, \lambda_{1}, \lambda_{2} \in(0, \infty),
$$

and for any $f_{1}, f_{2}: \mathbb{R}^{N} \rightarrow[0, \infty)$ continuous, convex functions satisfying (2.8).

\section{The Solution of HJB Equation}

An existence theorem for solutions of (3.19) is formally presented below presented.

Theorem 4.1. The system of equations (3.19) has a unique positive classical convex solution with quadratic growth, i.e.,

$$
z_{i}(x) \leq K_{i}\left(1+|x|^{2}\right), \text { for some } K_{i}>0, \quad i=1,2,
$$

and, such that

$$
\left|\nabla z_{i}(x)\right| \leq \bar{C}_{i}(1+|x|), \text { for } x \in \mathbb{R}^{N} \text { and for some positive constant } \bar{C}_{i} .
$$

We give a detailed proof of Theorem 4.1, which is based on the following two results.

Lemma 4.1. The system of partial differential equations with gradient term (3.19) is equivalent to the semilinear elliptic system

$$
\left\{\begin{array}{l}
\Delta u=u(x)\left[\frac{2}{k_{1}^{2}}\left(f_{1}(x)+\left(\lambda_{1}+a_{1}\right) k_{1} \ln u-a_{1} k_{2} \ln v\right)\right], \\
\Delta v=v(x)\left[\frac{2}{k_{2}^{2}}\left(f_{2}(x)+\left(\lambda_{2}+a_{2}\right) k_{2} \ln v-a_{2} k_{1} \ln u\right)\right],
\end{array} \quad x \in \mathbb{R}^{N} .\right.
$$

Proof. The change of variable

$$
z_{1}(x)=k_{1} w_{1}(x) \text { and } z_{2}(x)=k_{2} w_{2}(x),
$$

transform the system (3.19) into

$$
\left\{\begin{array}{l}
-\frac{k_{1}^{2}}{2} \Delta w_{1}+\frac{k_{1}^{2}\left|\nabla w_{1}\right|^{2}}{2}=f_{1}(x)-\left(\lambda_{1}+a_{1}\right) k_{1} w_{1}+a_{1} k_{2} w_{2}, \\
-\frac{k_{2}^{2}}{2} \Delta w_{2}+\frac{k_{2}^{2}\left|\nabla w_{2}\right|^{2}}{2}=f_{2}(x)-\left(\lambda_{2}+a_{2}\right) k_{1} w_{2}+a_{2} k_{1} w_{1},
\end{array}\right.
$$

or, equivalently

$$
\left\{\begin{array}{l}
-\Delta w_{1}+\left|\nabla w_{1}\right|^{2}=\frac{2}{k_{1}^{2}}\left[f_{1}(x)-\left(\lambda_{1}+a_{1}\right) k_{1} w_{1}+a_{1} k_{2} w_{2}\right], \\
-\Delta w_{2}+\left|\nabla w_{2}\right|^{2}=\frac{2}{k_{2}^{2}}\left[f_{2}(x)-\left(\lambda_{2}+a_{2}\right) k_{2} w_{2}+a_{2} k_{1} w_{1}\right] .
\end{array}\right.
$$

The change of variable

$$
u(x)=e^{-w_{1}(x)} \text { and } v(x)=e^{-w_{2}(x)},
$$

transform the system (4.24) into

$$
\left\{\begin{array}{l}
\Delta u=u\left[\frac{2}{k_{1}^{2}}\left(f_{1}(x)+\left(\lambda_{1}+a_{1}\right) k_{1} \ln u-a_{1} k_{2} \ln v\right)\right], \\
\Delta v=v\left[\frac{2}{k_{2}^{2}}\left(f_{2}(x)+\left(\lambda_{2}+a_{2}\right) k_{2} \ln v-a_{2} k_{1} \ln u\right)\right],
\end{array}\right.
$$

since

$$
\begin{aligned}
& \Delta u(x)=e^{-w_{1}(x)}\left(-\Delta w_{1}(x)+\left|\nabla w_{1}(x)\right|^{2}\right), \\
& \Delta v(x)=e^{-w_{2}(x)}\left(-\Delta w_{2}(x)+\left|\nabla w_{2}(x)\right|^{2}\right) .
\end{aligned}
$$

The existence of a solution $(u(x), v(x)) \in C^{2}\left(\mathbb{R}^{N}\right) \times C^{2}\left(\mathbb{R}^{N}\right)$ for the problem (4.22), such that $0<u(x) \leq 1$ and $0<v(x) \leq 1$, for all $x \in \mathbb{R}^{N}$, is proved in the following: 
Theorem 4.2. There exist functions $\underline{u}, \underline{v}, \bar{u}, \bar{v}: \mathbb{R}^{N} \rightarrow(0,1]$ of class $C^{2}\left(\mathbb{R}^{N}\right)$ such that

$$
\left\{\begin{array}{l}
-\Delta \underline{u}(x)+\underline{u}(x)\left[\frac{2}{k_{1}^{2}}\left(f_{1}(x)+\left(\lambda_{1}+a_{1}\right) k_{1} \ln \underline{u}(x)\right)\right] \leq 2 a_{1} \frac{k_{2}}{k_{1}^{2}} \underline{u}(x) \ln \underline{v}(x), \\
-\Delta \underline{v}(x)+\underline{v}(x)\left[\frac{2}{k_{2}^{2}}\left(f_{2}(x)+\left(\lambda_{2}+a_{2}\right) k_{2} \ln \underline{v}(x)\right)\right] \leq 2 a_{2} \frac{k_{1}}{k_{2}^{2}} \underline{v}(x) \ln \underline{u}(x), \\
-\Delta \bar{u}(x)+\bar{u}(x)\left[\frac{2}{k_{1}^{2}}\left(f_{1}(x)+\left(\lambda_{1}+a_{1}\right) k_{1} \ln \bar{u}(x)\right)\right] \geq 2 a_{1} \frac{k_{2}}{k_{1}^{2}} \bar{u}(x) \ln \bar{v}(x), \\
-\Delta \bar{v}(x)+\bar{v}(x)\left[\frac{2}{k_{2}^{2}}\left(f_{2}(x)+\left(\lambda_{2}+a_{2}\right) k_{2} \ln \bar{v}(x)\right)\right] \geq 2 a_{2} \frac{k_{1}}{k_{2}^{2}} \bar{v}(x) \ln \bar{u}(x), \\
\underline{u}(x) \leq \bar{u}(x), \underline{v}(x) \leq \bar{v}(x),
\end{array}\right.
$$

in the entire Euclidean space $\mathbb{R}^{N}$. In light of this the system (4.22) possesses an entire solution $(u, v) \in C^{2}\left(\mathbb{R}^{N}\right) \times C^{2}\left(\mathbb{R}^{N}\right)$ with $\underline{u}(x) \leq u(x) \leq \bar{u}(x)$ in $\mathbb{R}^{N}$ and $\underline{v}(x) \leq v(x) \leq \bar{v}(x)$ in $\mathbb{R}^{N}$.

Let us point out that the functions $(\underline{u}, \underline{v})$ (resp. $(\bar{u}, \bar{v})$ ) are called sub-solution (resp. super-solution) for the system (4.22).

Proof. In the following we construct the functions $(\underline{u}, \underline{v}),(\bar{u}, \bar{v})$ which satisfies the inequalities (4.22) in $\mathbb{R}^{N}$. We proceed as in Bensoussan, Sethi, Vickson and Derzko [2], for the scalar case. More exactly, we observe that there exist

$$
(\underline{u}(x), \underline{v}(x))=\left(e^{B_{1}|x|^{2}+D_{1}}, e^{B_{2}|x|^{2}+D_{2}}\right), \text { with } B_{1}, B_{2}, D_{1}, D_{2} \in(-\infty, 0),
$$

such that for all $\lambda_{1}>0$ and $\lambda_{2}>0$ the following hold

$$
\left\{\begin{array}{l}
-\Delta \underline{u}(x)+\underline{u}(x)\left[\frac{2}{k_{1}^{2}}\left(f_{1}(x)+\left(\lambda_{1}+a_{1}\right) k_{1} \ln \underline{u}(x)\right)\right] \leq 2 a_{1} \frac{k_{2}}{k_{1}^{2}} \underline{u}(x) \ln \underline{v}(x), \\
-\Delta \underline{v}(x)+\underline{v}(x)\left[\frac{2}{k_{2}^{2}}\left(f_{2}(x)+\left(\lambda_{2}+a_{2}\right) k_{2} \ln \underline{v}(x)\right)\right] \leq 2 a_{2} \frac{k_{1}}{k_{2}^{2}} \underline{v}(x) \ln \underline{u}(x),
\end{array}\right.
$$

i.e. $(\underline{u}(x), \underline{v}(x))$ is a sub-solution for the problem (4.22). Indeed, we find $B_{1}, B_{2}, D_{1}$, $D_{2} \in(-\infty, 0)$ such that the function $(\underline{u}(x), \underline{v}(x))$ defined (4.29) are solutions for (4.31)

$$
\left\{\begin{array}{l}
-\Delta \underline{u}(x)+\underline{u}(x)\left[\frac{2}{k_{1}^{2}}\left(M_{1}\left(|x|^{2}+1\right)+\left(\lambda_{1}+a_{1}\right) k_{1} \ln \underline{u}(x)\right)\right]=2 a_{1} \frac{k_{2}}{k_{1}^{2}} \underline{u}(x) \ln \underline{v}(x), \\
-\Delta \underline{v}(x)+\underline{v}(x)\left[\frac{2}{k_{2}^{2}}\left(M_{2}\left(|x|^{2}+1\right)+\left(\lambda_{2}+a_{2}\right) k_{2} \ln \underline{v}(x)\right)\right]=2 a_{2} \frac{k_{1}}{k_{2}^{2}} \underline{v}(x) \ln \underline{u}(x),
\end{array}\right.
$$

and, then sub-solution for for the problem (4.22). To do this, we write the system (4.31) in the equivalently form

$$
\left\{\begin{array}{l}
-2 B_{1}\left(2|x|^{2} B_{1}+1\right)-2 B_{1}(N-1)+\frac{2}{k_{1}^{2}}\left[M_{1}\left(|x|^{2}+1\right)+\left(\lambda_{1}+a_{1}\right) k_{1}\left(B_{1}|x|^{2}+D_{1}\right)\right] \\
=\frac{2 a_{1} k_{2}}{k_{1}^{2}}\left(B_{2}|x|^{2}+D_{2}\right), \\
-2 B_{2}\left(2|x|^{2} B_{2}+1\right)-2 B_{2}(N-1)+\frac{2}{k_{2}^{2}}\left[M_{2}\left(|x|^{2}+1\right)+\left(\lambda_{2}+a_{2}\right) k_{2}\left(B_{2}|x|^{2}+D_{2}\right)\right] \\
=\frac{2 a_{2} k_{1}}{k_{2}^{2}}\left(B_{1}|x|^{2}+D_{1}\right),
\end{array}\right.
$$

or, after rearranging the terms

$$
\left\{\begin{array}{l}
|x|^{2}\left[-4 B_{1}^{2}+\frac{2 M_{1}}{k_{1}^{2}}+\frac{2}{k_{1}}\left(\lambda_{1}+a_{1}\right) B_{1}-2 a_{1} \frac{k_{2}}{k_{1}^{2}} B_{2}\right]-2 B_{1} N \\
+\frac{2 M_{1}}{k_{1}^{2}}+\frac{2}{k_{1}}\left(\lambda_{1}+a_{1}\right) D_{1}-\frac{2 a_{1} k_{2} D_{2}}{k_{1}^{2}}=0 \\
|x|^{2}\left[-4 B_{2}^{2}+\frac{2 M_{2}}{k_{2}^{2}}+\frac{2}{k_{2}}\left(\lambda_{2}+a_{2}\right) B_{2}-2 a_{2} \frac{k_{1}}{k_{2}^{2}} B_{1}\right]-2 B_{2} N \\
+\frac{2 M_{2}}{k_{2}^{2}}+\frac{2}{k_{2}}\left(\lambda_{2}+a_{2}\right) D_{2}-\frac{2 a_{2} k_{1} D_{1}}{k_{2}^{2}}=0 .
\end{array}\right.
$$


Now, we consider the system of equations

$$
\left\{\begin{array}{l}
-4 B_{1}^{2}+\frac{2 M_{1}}{k_{1}^{2}}+\frac{2}{k_{1}}\left(\lambda_{1}+a_{1}\right) B_{1}-2 a_{1} \frac{k_{2}}{k_{1}^{2}} B_{2}=0 \\
-2 B_{1} N+\frac{2 M_{1}}{k_{1}^{2}}+\frac{2}{k_{1}}\left(\lambda_{1}+a_{1}\right) D_{1}-2 a_{1} \frac{k_{2}}{k_{1}^{2}} D_{2}=0 \\
-4 B_{2}^{2}+\frac{2 M_{2}}{k_{2}^{2}}+\frac{2}{k_{2}}\left(\lambda_{2}+a_{2}\right) B_{2}-2 a_{2} \frac{k_{1}}{k_{2}^{2}} B_{1}=0 \\
-2 B_{2} N+\frac{2 M_{2}}{k_{2}^{2}}+\frac{2}{k_{2}}\left(\lambda_{2}+a_{2}\right) D_{2}-2 a_{2} \frac{k_{1}}{k_{2}^{2}} D_{1}=0
\end{array}\right.
$$

Since we wish to analyze the existence of $B_{1}, B_{2}, D_{1}, D_{2} \in(-\infty, 0)$ that solve (4.32) we couple the Equations 1 and 3 together

$$
\left(\begin{array}{c}
4 B_{1}^{2}-\frac{2 M_{1}}{k_{1}^{2}} \\
4 B_{2}^{2}-\frac{2 M_{2}}{k_{2}^{2}}
\end{array}\right)=\left(\begin{array}{cc}
\frac{2}{k_{1}}\left(\lambda_{1}+a_{1}\right) & -2 a_{1} \frac{k_{2}}{k_{1}^{2}} \\
-2 a_{2} \frac{k_{1}}{k_{2}^{2}} & \frac{2}{k_{2}}\left(\lambda_{2}+a_{2}\right)
\end{array}\right)\left(\begin{array}{c}
B_{1} \\
B_{2}
\end{array}\right)
$$

and, similarly for the Equations 2 and 4

$$
\left(\begin{array}{c}
2 B_{1} N \\
2 B_{2} N
\end{array}\right)=\left(\begin{array}{cc}
\frac{2}{k_{1}}\left(\lambda_{1}+a_{1}\right) & -2 a_{1} \frac{k_{2}}{k_{1}^{2}} \\
-2 a_{2} \frac{k_{1}}{k_{2}^{2}} & \frac{2}{k_{2}}\left(\lambda_{2}+a_{2}\right)
\end{array}\right)\left(\begin{array}{c}
D_{1} \\
D_{2}
\end{array}\right)
$$

Clearly

$$
\left|\begin{array}{cc}
\frac{2}{k_{1}}\left(\lambda_{1}+a_{1}\right) & -2 a_{1} \frac{k_{2}}{k_{1}^{2}} \\
-2 a_{2} \frac{k_{1}}{k_{2}^{2}} & \frac{2}{k_{2}}\left(\lambda_{2}+a_{2}\right)
\end{array}\right|=\frac{1}{k_{1} k_{2}}\left(4 \lambda_{1} \lambda_{2}+4 \lambda_{1} a_{2}+4 \lambda_{2} a_{1}\right)>0,
$$

and, so the system (4.33) can be written equivalently as

$$
\left(\begin{array}{l}
-B_{1} \\
-B_{2}
\end{array}\right)=\frac{1}{2 \lambda_{1} \lambda_{2}+2 \lambda_{1} a_{2}+2 \lambda_{2} a_{1}}\left(\begin{array}{cc}
k_{1}\left(\lambda_{2}+a_{2}\right) & \frac{a_{1} k_{2}^{2}}{k_{1}} \\
\frac{a_{2} k_{1}^{2}}{k_{2}} & k_{2}\left(a_{1}+\lambda_{1}\right)
\end{array}\right)\left(\begin{array}{c}
\frac{2 M_{1}}{k_{1}^{2}}-4 B_{1}^{2} \\
\frac{2 M_{2}}{k_{2}^{2}}-4 B_{2}^{2}
\end{array}\right) .
$$

The existence of $B_{1}, B_{2} \in(-\infty, 0)$ can be easy proved by observing that the continuous functions $h_{1}, h_{2}:(-\infty, 0] \rightarrow \mathbb{R}$ defined by

$$
\begin{aligned}
& h_{1}\left(B_{1}\right)=-4 B_{1}^{2}+\frac{2 M_{1}}{k_{1}^{2}}+\frac{2\left(\lambda_{1}+a_{1}\right) B_{1}}{k_{1}}-\frac{a_{1}}{2 k_{1}^{2}}\left(\lambda_{2}+a_{2}-\sqrt{\lambda_{2}^{2}+2 \lambda_{2} a_{2}+a_{2}^{2}-8 B_{1} k_{1} a_{2}+8 M_{2}}\right), \\
& h_{2}\left(B_{2}\right)=-4 B_{2}^{2}+\frac{2 M_{2}}{k_{2}^{2}}+\frac{2\left(\lambda_{2}+a_{2}\right) B_{2}}{k_{2}}-\frac{a_{2}}{2 k_{2}^{2}}\left(\lambda_{1}+a_{1}-\sqrt{\lambda_{1}^{2}+2 \lambda_{1} a_{1}+a_{1}^{2}-8 B_{2} k_{2} a_{1}+8 M_{1}}\right),
\end{aligned}
$$

has the following properties

$$
h_{1}(-\infty)=-\infty \text { and } h_{2}(-\infty)=-\infty
$$

respectively

$$
\begin{aligned}
& h_{1}(0)=\frac{2 M_{1}}{k_{1}^{2}}-\frac{a_{1}}{2 k_{1}^{2}}\left(\lambda_{2}+a_{2}-\sqrt{\lambda_{2}^{2}+2 \lambda_{2} a_{2}+a_{2}^{2}+8 M_{2}}\right)>0, \\
& h_{2}(0)=\frac{2 M_{2}}{k_{2}^{2}}-\frac{a_{2}}{2 k_{2}^{2}}\left(\lambda_{1}+a_{1}-\sqrt{\lambda_{1}^{2}+2 \lambda_{1} a_{1}+a_{1}^{2}+8 M_{1}}\right)>0 .
\end{aligned}
$$

The observations (4.36) and (4.37) say that the equation

$$
\left(h_{1}\left(B_{1}\right), h_{2}\left(B_{2}\right)\right)=(0,0) \text {, }
$$

has at least one solution in $(-\infty, 0) \times(-\infty, 0)$ and furthermore it is unique (see also, the references $[18,19])$. Thus, there exist and are unique $B_{1}, B_{2} \in(-\infty, 0)$ that solve the system of equations (4.35).

Next, we observe that the system (4.34) can be written equivalently as

$$
\left(\begin{array}{c}
D_{1} \\
D_{2}
\end{array}\right)=\left(\begin{array}{cc}
\frac{\lambda_{2} k_{1}+a_{2} k_{1}}{2 \lambda_{1} \lambda_{2}+2 \lambda_{1} a_{2}+2 \lambda_{2} a_{1}} & a_{1} \frac{k_{2}^{2}}{2 \lambda_{1} \lambda_{2} k_{1}+2 \lambda_{1} a_{2} k_{1}+2 \lambda_{2} a_{1} k_{1}} \\
a_{2} \frac{a_{1}^{2} k_{2}^{2}+\lambda_{1} k_{2}^{2}}{2 \lambda_{1} \lambda_{2} k_{2}+2 \lambda_{1} a_{2} k_{2}+2 \lambda_{2} a_{1} k_{2}} & \frac{a_{1}}{2 \lambda_{1} \lambda_{2} k_{2}+2 \lambda_{1} a_{2} k_{2}+2 \lambda_{2} a_{1} k_{2}}
\end{array}\right)\left(\begin{array}{c}
2 B_{1} N \\
2 B_{2} N
\end{array}\right),
$$


from where we can see that there exist $B_{1}, B_{2}, D_{1}, D_{2} \in(-\infty, 0)$ that solve (4.32) and then $(\underline{u}(x), \underline{v}(x))$ are such that the inequalities in (4.30) hold.

To construct a super-solution it is useful to remember that $\ln 1=0$ and then a simple calculation shows that

is a super-solution of the problem (4.22).

$$
(\bar{u}(x), \bar{v}(x))=(1,1),
$$

Until now, we constructed the corresponding sub- and super-solutions employed in the scalar case by [2]. Clearly, (4.28) holds and then in Theorem 4.2 it remains to prove that there exists $(u(x), v(x)) \in C^{2}\left(\mathbb{R}^{N}\right) \times C^{2}\left(\mathbb{R}^{N}\right)$ with $\underline{u}(x) \leq u(x) \leq \bar{u}(x)$ in $\mathbb{R}^{N}$ and $\underline{v}(x) \leq v(x) \leq \bar{v}(x)$ in $\mathbb{R}^{N}$ satisfying (4.22).

To do this, let $B_{k}$ be the ball whose center is the origin of $\mathbb{R}^{N}$ and which has radius $k=1,2, \ldots$. We consider the boundary value problem

$$
\left\{\begin{array}{l}
\Delta u=u\left[\frac{2}{k_{1}^{2}}\left(f_{1}(x)+\left(\lambda_{1}+a_{1}\right) k_{1} \ln u-a_{1} k_{2} \ln v\right)\right], x \in B_{k}, \\
\Delta v=v\left[\frac{2}{k_{2}^{2}}\left(f_{2}(x)+\left(\lambda_{2}+a_{2}\right) k_{2} \ln v-a_{2} k_{1} \ln u\right)\right], x \in B_{k}, \\
u(x)=\underline{u}_{k}(x), v(x)=\underline{v}_{k}(x), x \in \partial B_{k},
\end{array}\right.
$$

where $\underline{u}_{k}=\underline{u}_{\mid B_{k}}$ and $\underline{v}_{k}=\underline{v}_{\mid B_{k}}$. In a similar way, we define $\bar{u}_{k}=\bar{u}_{\mid B_{k}}$ and $\bar{v}_{k}=\bar{v}_{\mid B_{k}}$ then $\underline{u}_{k}, \bar{u}_{k}, \underline{v}_{k}, \bar{v}_{k} \in C^{2}\left(\bar{B}_{k}\right)$.

Observing that

$$
\begin{aligned}
\inf _{x \in \mathbb{R}^{N}} \underline{u}(x) & \leq \min _{x \in \bar{B}_{k}} \underline{u}_{k}(x) \text { and } \sup _{x \in \mathbb{R}^{N}} \bar{u}(x) \geq \max _{x \in \bar{B}_{k}} \bar{u}_{k}(x), \\
\inf _{x \in \mathbb{R}^{N^{N}}} \underline{v}(x) & \leq \min _{x \in \bar{B}_{k}} \underline{v}_{k}(x) \text { and } \sup _{x \in \mathbb{R}^{N}} \bar{v}(x) \geq \max _{x \in \bar{B}_{k}} \bar{v}_{k}(x),
\end{aligned}
$$

a result of Reis Gaete [16] (see also the pioneering papers of Kawano [23] and Lee, Shivaji and Ye [25]), proves the existence of a solution $\left(u_{k}, v_{k}\right) \in\left[C^{2}\left(B_{k}\right) \cap C\left(\bar{B}_{k}\right)\right]^{2}$ satisfying the system (4.38). The functions $\left(u_{k}, v_{k}\right)$ also satisfy

$$
\begin{aligned}
& \underline{u}_{k}(x) \leq u_{k}(x) \leq \bar{u}_{k}(x), x \in \bar{B}_{k}, \\
& \underline{v}_{k}(x) \leq v_{k}(x) \leq \bar{v}_{k}(x), x \in \bar{B}_{k} .
\end{aligned}
$$

By a standard regularity argument based on Schauder estimates, see Tolksdorf [31, 17, proposition 3.7, p. 806] and Reis Gaete [16] for details, we can see that for all integers $k \geq n+1$ there are $\alpha_{1}, \alpha_{2} \in(0,1)$ and positive constants $C_{1}, C_{2}$, independent of $k$ such that

$$
\left\{\begin{array}{l}
u_{k} \in C^{2, \alpha_{1}}\left(\bar{B}_{n}\right) \text { and }\left|u_{k}\right|_{C^{2, \alpha_{1}}\left(\bar{B}_{n}\right)}<C_{1}, \\
v_{k} \in C^{2, \alpha_{2}}\left(\bar{B}_{n}\right) \text { and }\left|v_{k}\right|_{C^{2, \alpha_{2}}\left(\bar{B}_{n}\right)}<C_{2},
\end{array}\right.
$$

where $|\circ|_{C^{2, \circ}}$ is the usual norm of the space $C^{2, \circ}\left(\bar{B}_{n}\right)$. Moreover, there exist constants: $C_{3}$ independent of $u_{k}, C_{4}$ independent of $v_{k}$ and such that

$$
\left\{\begin{array}{l}
\max _{x \in \bar{B}_{n}}\left|\nabla u_{k}(x)\right| \leq C_{3} \max _{x \in \bar{B}_{k}}\left|u_{k}(x)\right|, \\
\max _{x \in \bar{B}_{n}}\left|\nabla v_{k}(x)\right| \leq C_{4} \max _{x \in \bar{B}_{k}}\left|v_{k}(x)\right| .
\end{array}\right.
$$

The information from (4.39) and (4.40) implies that $\left\{\left(\nabla u_{k}, \nabla v_{k}\right)\right\}_{k}$ as well as $\left\{\left(u_{k}, v_{k}\right)\right\}_{k}$ are uniformly bounded on $\bar{B}_{n}$. We wish to show that this sequence $\left\{\left(u_{k}, v_{k}\right)\right\}_{k}$ contains a subsequence converging to a desired entire solution of (4.22). Next, we concentrate our attention to the sequence $\left\{u_{k}\right\}_{k}$. Using the compactness of the embedding $C^{2, \alpha_{1}}\left(\bar{B}_{n}\right) \hookrightarrow$ $C^{2}\left(\bar{B}_{n}\right)$, enables us to define the subsequence

$$
u_{n}^{k}:=u_{k \mid B_{n}}, \text { for all } k \geq n+1 .
$$


Then for $n=1,2,3, \ldots$ there exist a subsequence $\left\{u_{n}^{k_{n j}}\right\}_{k \geq n+1, j \geq 1}$ of $\left\{u_{n}^{k}\right\}_{k \geq n+1}$ and a function $u_{n}$ such that

$$
u_{n}^{k_{n j}} \rightarrow u_{n}
$$

uniformly in the $C^{2}\left(\bar{B}_{n}\right)$ norm. More exactly, we get through a well-known diagonal process that

$$
\begin{aligned}
& \mathbf{u}_{1}^{k_{11}}, u_{1}^{k_{12}}, u_{1}^{k_{13}}, \ldots \quad \longrightarrow \quad u_{1} \text { in } C^{2}\left(\bar{B}_{1}\right) \text {, } \\
& u_{2}^{k_{21}}, \mathbf{u}_{2}^{k_{22}}, u_{2}^{k_{23}}, \ldots \rightarrow u_{2} \text { in } C^{2}\left(\bar{B}_{2}\right) \text {, } \\
& u_{3}^{k_{31}}, u_{3}^{k_{32}}, \mathbf{u}_{3}^{k_{33}}, \ldots \quad \longrightarrow u_{3} \text { in } C^{2}\left(\bar{B}_{3}\right) \text {, }
\end{aligned}
$$

Since $\mathbb{R}^{N}=\bigcup_{n=1}^{\infty} B_{n}$, we can define the function $u: \mathbb{R}^{N} \rightarrow[0, \infty)$ such that

$$
u(x)=\lim _{n \rightarrow \infty} u_{n}(x) .
$$

Let us give the construction of the function $u$ for the problem (4.22). This is obtained by considering the sequence $\left(u_{d}^{k_{d d}}\right)_{d \geq 1}$ and the sequence $\left(u_{n}^{k_{n d}}\right)_{k \geq n+1}$, restricted to the ball $B_{n}$, which are such that

$$
u_{n}^{k_{n d}} \stackrel{d \rightarrow \infty}{\rightarrow} u_{n}:=u(x) \text { for all } x \in B_{n},
$$

and then, for $d \rightarrow \infty$ we obtain

$$
u_{d}^{k_{d d}} \stackrel{d \rightarrow \infty}{\rightarrow} u(x) \text { in } C^{2}\left(\mathbb{R}^{N}\right),
$$

according with the diagonal process. Furthermore, since

$$
\underline{u}(x) \leq u_{d}^{k_{d d}} \leq \bar{u}(x), \text { for } x \in \mathbb{R}^{N},
$$

and for each $d=1,2,3, \ldots$ the following relation is valid

$$
\underline{u}(x) \leq u(x) \leq \bar{u}(x), \text { for } x \in \mathbb{R}^{N} .
$$

We employ the same iteration scheme to construct the function $v: \mathbb{R}^{N} \rightarrow[0, \infty)$ such that

$$
v(x)=\lim _{n \rightarrow \infty} v_{n}(x) .
$$

From the regularity theory the solution $(u, v)$ belongs to $C^{2}\left(\mathbb{R}^{N}\right) \times C^{2}\left(\mathbb{R}^{N}\right)$ and satisfies (4.22). This completes the proof of Theorem 4.2.

Proof of Theorem 4.1. As easily verified, the existence of solutions is proved by Lemma 4.1 and Theorem 4.2. Then it remains to prove (4.20).

A recapitulation of the changes of variables says that

$$
z_{1}(x)=-k_{1} \ln u(x) \text { and } z_{2}(x)=-k_{2} \ln v(x),
$$

is a solution for (3.19). Observing that

$$
\underline{u}(x)=e^{B_{1}|x|^{2}+D_{1}} \leq u(x) \leq \bar{u}(x)=1, x \in \mathbb{R}^{N},
$$

it follows that

$$
B_{1}|x|^{2}+D_{1} \leq \ln u(x) \leq \ln 1,
$$

and then

or equivalently

$$
0 \leq-k_{1} \ln u(x) \leq-k_{1} B_{1}|x|^{2}-k_{1} D_{1}
$$

$$
0 \leq z_{1}(x) \leq K_{1}\left(|x|^{2}+1\right), \text { for } x \in \mathbb{R}^{N} \text { and } K_{1}=\max \left\{-k_{1} B_{1},-k_{1} D_{1}\right\} .
$$


In the same way

$$
0 \leq z_{2}(x) \leq K_{2}\left(|x|^{2}+1\right), \text { for } x \in \mathbb{R}^{N} \text { and } K_{2}=\max \left\{-k_{2} B_{2},-k_{2} D_{2}\right\},
$$

and the proof is completed.

By classical arguments the solution $\left(z_{1}(x), z_{2}(x)\right)$ is convex. Since $\left(z_{1}(x), z_{2}(x)\right)$ verifies (4.20) the inequality (4.21) follows from [14, Lemma 1, p. 24] (see also the arguments in [15, Theorem 1, p. 236]). The uniqueness of such a solution follows from the result of $[13,22]$ (see also the former papers of $[20,21])$, since for our system their comparison results can also be set in $\mathbb{R}^{N}$, instead of a domain $\Omega \subset \mathbb{R}^{N}$. The proof is completed.

\section{VERIFICATION}

In this section we establish the optimality of control

$$
\hat{c}_{i}(x)=\nabla u(x, i)=-\nabla z_{i}(x) .
$$

Its associated Markov modulated diffusion is

$$
d X^{i}(t)=\hat{c}_{\epsilon(t)}^{i}(X(t)) d t+k_{\epsilon(t)} d W^{i}(t), i=1, \ldots N .
$$

The verification theorem proceeds with the following steps:

First Step: To establish the solution for SDE (5.44) one can apply the result of Section 2 in [26]; since one of the conditions is satisfied in light of (4.21), it only remains to check for the locally Lipschitz property of $x \rightarrow \nabla z_{i}(x)$. According to [27] Lemma 1.2.3, the Lipschitz property of convex functions' gradient is equivalent to a quadratic upper bound on the function. Thus, in light of (4.20), the locally Lipschitz property of $x \rightarrow \nabla z_{i}(x)$ yields, so SDE (5.44) has a unique solution.

Second Step: Let $X(t)$ be the solution of (5.44). In light of (4.21) one can get using exercise 7.5 of [28] that

$$
E|X(t)|^{2} \leq C_{1} e^{C_{2} t}
$$

for some positive constants $C_{1}, C_{2}$.

Third Step: The set of acceptable controls that we consider is encompassing of controls $c$ for which

$$
J(x, c, i)=E\left[\int_{0}^{\infty} e^{-\lambda_{\epsilon(t)} t}\left[f_{\epsilon(t)}(X(t))+\frac{1}{2}|c|_{\epsilon(t)}^{2}(X(t))\right] d t \mid X(0)=x, \epsilon(0)=i\right]<\infty,
$$

and the following transversality condition

$$
\lim _{t \rightarrow \infty} E e^{-\lambda_{\epsilon(t)} t}|X(t)|^{2}=0,
$$

is met. Because of (4.21), estimates (4.20), (5.45), the candidate optimal control $\hat{c}$ of (5.43) verifies that $J(x, c, i)<\infty$, for $\lambda_{1}, \lambda_{2}$ large enough. Moreover, there exist $\lambda_{1}>0$ and $\lambda_{2}>0$ large enough such that the transversality condition (3.11) is met because of (4.20) and (5.45). Also the control $c=0$, is an acceptable control.

In light of the quadratic estimate on the value function (see (4.20) in theorem 2.1), the transversality condition implies that

$$
\lim _{t \rightarrow \infty} E e^{-\lambda_{\epsilon(t)} t} u(X(t), \epsilon(t))=0 .
$$

Fourth Step: Recall that

$$
M^{c}(t)=e^{-\lambda_{\epsilon(t)} t} u(X(t), \epsilon(t))-\int_{0}^{t} e^{-\lambda_{\epsilon(u)} u}\left[f_{\epsilon(t)}(X(u))+\frac{1}{2}|c|_{\epsilon(u)}^{2}(X(u))\right] d u .
$$


Therefore, the Itô's Lemma yields for the optimal control candidate, $\hat{c}$

$$
d M^{c}(t)=e^{-\lambda_{\epsilon(t)} t} k_{\epsilon(t)} \nabla u(X(t), \epsilon(t)) d W(t) .
$$

Consequently $M^{\hat{c}}(t)$ is a local martingale. Moreover, for $\lambda_{1}, \lambda_{2}$ large enough, in light of (4.21), and (5.45),

$$
E \int_{0}^{t} e^{-2 \lambda_{\epsilon(s)} s} k_{\epsilon(s)}^{2}|\nabla u(X(s), \epsilon(s))|^{2} d s \leq \bar{C},
$$

for some positive constants $\bar{C}$. This in turn makes $M^{\hat{c}}(t)$ a (true) martingale.

Fifth Step: This step establishes the optimality of $\hat{c}$ of (5.43). The HJB equation (3.16) is equivalent to

$$
\sup _{c} L^{c} u(x, i)=0, \quad L^{\hat{c}} u(x, i)=0, i=1,2 .
$$

The martingale/supermartingale principle yields

$$
E e^{-\lambda_{\epsilon(t)} t} u(X(t), \epsilon(t))-E \int_{0}^{t} e^{-\lambda_{\epsilon(u)} u}\left[f_{\epsilon(t)}(X(u))+\frac{1}{2}|\hat{c}|_{\epsilon(u)}^{2}(X(u))\right] d u=u(x, \epsilon(0)),
$$

and

$$
E e^{-\lambda_{\epsilon(t)} t} u(X(t), \epsilon(t))-E \int_{0}^{t} e^{-\lambda_{\epsilon(u)} u}\left[f_{\epsilon(t)}(X(u))+\frac{1}{2}|c|_{\epsilon(u)}^{2}(X(u))\right] d u \leq u(x, \epsilon(0)) .
$$

By passing $t \rightarrow \infty$ and using transversality condition (5.47) we get the optimality of $\hat{c}$.

\section{A FIXED POINT CHARACTERIZATION OF OPTIMAL CONTROLS}

6.1. The subgame perfect controls. For a controll $\{c(t)\}_{t \geq 0}$ and its corresponding state process $\{X(t)\}_{t \geq 0}$ given by (2.6), we follow [11] to give a rigorous mathematical formulation of the subgame perfect production controls in the formal definition below.

Definition 6.1. Let $F=\left(F_{i}, i=1, \ldots N\right): \mathbb{R} \times\{1,2\} \rightarrow \mathbb{R}^{N}$ be a vector map such that for any $x>0$ and $i \in\{1,2\}$

$$
\liminf \frac{J(x, \bar{c}, i)-J\left(x, c_{\epsilon}, i\right)}{\epsilon} \leq 0,
$$

where the subgame perfect controls

$$
\bar{c}(s) \triangleq F(\bar{X}(s), \epsilon(s)) .
$$

Here, the process $\{\bar{X}(s)\}_{s \geq 0}$ is the state process corresponding to $\{\bar{c}(s)\}_{s \geq 0}$. The control $\left\{c_{\epsilon}(s)\right\}_{s \geq 0}$ is defined by

$$
c_{\epsilon}(s)= \begin{cases}\bar{c}(s), & s \in[0, \infty] \backslash E_{\epsilon, 0} \\ c(s), & s \in E_{\epsilon, 0},\end{cases}
$$

with $E_{\epsilon, 0}=[0, \epsilon] ;\{c(s)\}_{s \in E_{\epsilon, 0}}$ is any control. If (6.49) holds true, then $\{\bar{c}(s)\}_{s \geq 0}$ is a subgame perfect control.

Let us remark that the optimal control

$$
\hat{c}_{i}(x)=\nabla u(x, i)=-\nabla z_{i}(x),
$$

given by (3.17) of the previous section is a subgame perfect control with

$$
F(x, i) \triangleq-\nabla z_{i}(x)
$$

since

$$
\hat{c}_{i}=\arg \min _{c} J(x, \bar{c}, i)
$$


and thus (6.49) is automatically satisfied.

6.2. The value function and the generalized HJB equation. Inspired by [11], the value function $z_{i}: \mathbb{R}^{N} \times\{1,2\} \rightarrow \mathbb{R}$ is a $C^{2}$ function, convex defined by

$$
z_{i}(x) \triangleq E\left[\int_{0}^{\infty} e^{-\lambda_{\epsilon(t)} t}\left[f_{\epsilon(t)}(\bar{X}(t))+\frac{1}{2}|F(\bar{X}(t), \epsilon(t))|^{2}\right] d t \mid \epsilon(0)=i\right]
$$

Recall that $\{\bar{X}(s)\}_{s \geq 0}$ is the state process corresponding to $\{\bar{c}(s)\}_{s \geq 0}$, and given by (2.6). Moreover, take

$$
F(x, i) \triangleq-\nabla z_{i}(x), i \in\{1,2\}
$$

The following system of equations (6.52), (2.6), and (6.53) are called generalized HJB equation. It can be shown as in [11] that a solution $z_{i}(x), i \in\{1,2\}$ to this generalized HJB equation leads to a subgame perfect control through (6.53). The generalized HJB equation is a fixed point type equation (6.52), coupled with a Hamiltonian condition, (6.53), and a stochastic flow equation, (2.6). The fixed point equation (6.52) defined the value function as the continuation cost. There is an economical interpretation of this; if the decision maker implements the control according to (6.52), given the current time, state $i$ of the Markov chain, and inventory $x$, and evaluates the expected cost criterion, then this will turn out to be exactly $z_{i}(x)$,

Next we show that under $C^{1,2}$ differentiability assumption, the solution of the generalized HJB equation solves the HJB equation as well. Indeed, the process

$$
M^{F}(t)=e^{-\lambda_{\epsilon(t)}} z_{\epsilon(t)}(X(t))-\int_{0}^{t} e^{-\lambda_{\epsilon(u)} u}\left[f_{\epsilon(t)}(\bar{X}(u))+\left.\frac{1}{2} F(\bar{X}(u), \epsilon(u))\right|^{2} d u .\right.
$$

is a martingale. By applying Itô's Lemma and setting the drift to 0 we get that $z_{i}(x)$, $(i=1,2)$ solve (3.19). Conversely the solution of the HJB equation together with the transversality condition makes the process in (6.54) a martingale (choosing $F$ as in (6.52)). The martingale property and the transversality condition yields (6.53) whence the generalized HJB equation.

Therefore $z_{i}(x), i=1,2$ of (3.19) admits a fixed point characterization through equations (6.52) and (6.53).

\section{SPECIAL CASE}

In the following we manage to obtain a simple closed form solution for our system given a special loss functions of the type $f_{1}(x)=f_{2}(x)=|x|^{2}$. That is, assume

$$
\left\{\begin{array}{l}
\Delta u=u(x)\left[\frac{2}{k_{1}^{2}}\left(|x|^{2}+\left(\lambda_{1}+a_{1}\right) k_{1} \ln u-a_{1} k_{2} \ln v\right)\right], \\
\Delta v=v(x)\left[\frac{2}{k_{2}^{2}}\left(|x|^{2}+\left(\lambda_{2}+a_{2}\right) k_{2} \ln v-a_{2} k_{1} \ln u\right)\right],
\end{array} \quad x \in \mathbb{R}^{N} .\right.
$$

Then, by the same arguments used for (4.32), the unique solution for the problem (7.55) is

$$
(u(x), v(x))=\left(e^{B_{1}|x|^{2}+D_{1}}, e^{B_{2}|x|^{2}+D_{2}}\right),
$$

with $B_{1}, B_{2}, D_{1}, D_{2} \in(-\infty, 0)$ solving the elementary system of nonlinear equations

$$
\left\{\begin{array}{l}
-4 B_{1}^{2}+\frac{2}{k_{1}^{2}}+\frac{2}{k_{1}}\left(\lambda_{1}+a_{1}\right) B_{1}-2 a_{1} \frac{k_{2}}{k_{1}^{2}} B_{2}=0 \\
-2 B_{1} N+\frac{2}{k_{1}}\left(\lambda_{1}+a_{1}\right) D_{1}-2 a_{1} \frac{k_{2}}{k_{1}^{2}} D_{2}=0 \\
-4 B_{2}^{2}+\frac{2}{k_{2}^{2}}+\frac{2}{k_{2}}\left(\lambda_{2}+a_{2}\right) B_{2}-2 a_{2} \frac{k_{1}}{k_{2}^{2}} B_{1}=0 \\
-2 B_{2} N+\frac{2}{k_{2}}\left(\lambda_{2}+a_{2}\right) D_{2}-2 a_{2} \frac{k_{1}}{k_{2}^{2}} D_{1}=0,
\end{array}\right.
$$


for which we know there exists a unique solution in light of Theorem 4.2. Let us point out that (4.25) implies

$$
z_{1}(x)=-k_{1}\left(B_{1}|x|^{2}+D_{1}\right)>0 \text { and } z_{2}(x)=-k_{2}\left(B_{2}|x|^{2}+D_{2}\right)>0 \text { for all } x \in \mathbb{R}^{N},
$$

i.e. $\left(z_{1}(x), z_{2}(x)\right)$ is the positive solution obtained with the above procedure. For the stochastic control problem we choose the positive solution, i.e., the one given in (7.57).

Let us provide an lower bound estimate for $\lambda_{1}, \lambda_{2}$ in this special case, so that transversality condition

$$
\lim _{t \rightarrow \infty} E\left[e^{-\lambda_{\epsilon(t)}}|X(t)|^{2}\right]=0,
$$

holds true. The SDE system (5.44) in this case becomes

$$
d X^{i}(t)=2 k_{\epsilon(t)} B_{\epsilon(t)} X^{i}(t) d t+k_{\epsilon(t)} d W^{i}(t), i=1, \ldots N .
$$

By applying Itô's Lemma one gets

$$
\begin{aligned}
d\left(X^{i}(t)\right)^{2} & =2 X^{i}(t) d X^{i}(t)+d X^{i}(t) d X^{i}(t) \\
& =\left[2 k_{\epsilon(t)} B_{\epsilon(t)}\left(X^{i}(t)\right)^{2}+k_{\epsilon(t)}^{2}\right] d t+2 X^{i}(t) k_{\epsilon(t)} d W^{i}(t) .
\end{aligned}
$$

Let us denote $F_{i}(t)=E\left[\left(X^{i}(t)\right)^{2}\right]$. By taking expectations in the above equation we get

$$
F_{i}(t)=E\left[\int_{0}^{t}\left[2 k_{\epsilon(s)} B_{\epsilon(s)}\left(X^{i}(s)\right)^{2}+k_{\epsilon(s)}^{2}\right] d s\right]+\left(X^{i}(0)\right)^{2} .
$$

Then, in the light of the above equation and negativity of $B_{1}, B_{2}$, we get that $F_{i}(t)$ has linear growth. Therefore, the transversality condition to holds true.

In summary, in this article we have reduced the stochastic production planning problem with regime switching in the economy to the demonstration of the existence of a unique solution to a system of partial differential equations.

Acknowledgments. Traian A. Pirvu acknowledges that this work was supported by NSERC grant 5-36700.

\section{REFERENCES}

[1] Alvarez, O. A quasilinear elliptic equation in $\mathbb{R}^{N}$. Proc. Roy. Soc Edinburgh Sect A, 126 (1996), 911-921.

[2] Bensoussan, A.; Sethi, S. P.; Vickson, R.; Derzko, N. Stochastic production planning with production constraints. SIAM J. Control Optim. 22 (1984), 920-935.

[3] Cadenilas, A.; Lakner, P.; Pinedo, M. Optimal production management when demand depends on the business cycle. Operations Research 61 (2013), no. 4, 1046-1062

[4] Canepa, E. C.; Covei, D. P.; Pirvu, T. A. A Stochastic production planning problem. Fixed Point Theory forthcoming.

[5] Canepa, E. C.; Covei, D. P.; Pirvu, T. A. Stochastic production planning with regime switching. Acta Mathematica Scientia forthcoming. https://arxiv.org/abs/2002.09724

[6] Covei, D.-P. Entire Solutions of a Nonlinear Diffusion System. Chapter Book in Applied Mathematical Analysis: Theory, Methods, and Applications, Studies in Systems, Decision and Control (H. Dutta, J. F. Peters Editors), Springer Nature Switzerland AG 2020.

[7] Covei, D.-P.; Pirvu, T. A. An elliptic partial differential equation and its application. Appl. Math. Lett. 101 (2020), 1-7.

[8] Covei, D.-P., A remark on the radial solutions of a modified Schrodinger system by the dual approach. Math. Commun. 24 (2019), 245-263.

[9] Covei, D.-P. The Keller-Osserman-type conditions for the study of a semilinear elliptic system. Bound. Value Probl., 104 (2019), 1-14

[10] Covei, D.-P., Symmetric solutions for an elliptic partial differential equation that arises in stochastic production planning with production constraints. Appl. Math. Comput. 350 (2019), 190-197.

[11] Ekeland, I.; Pirvu, T. A. Investment and consumption without commitment. Mathematics and Financial Economics 2 (2008), 57-86.

[12] Ekeland, I.; Mbodji, O.; Pirvu, T. A. Time consistent portfolio management. Siam J. Financial Math. 3 (2010), $1-32$. 
[13] Engler, H.; Lenhart, S. M. Viscosity Solutions for Weakly Coupled Systems of Hamilton-Jacobi Equations. Proc. London Math. Soc. 63 (1991), No. 3, 212-240.

[14] Evans, L. C. Weak Convergence Methods for Nonlinear Partial Differential Equations. CBMS Regional Conference Series in Mathematics, 74 (1990), 1-82.

[15] Evans, L. C.; Gariepy, R. F. Measure Theory and Fine Properties of Functions. Chapman and Hall/CRC Published April 14, 2015 Textbook - 313 Pages.

[16] Gaete, M. R. Reis. Soluçöes positivas para sistemas elí pticos quasilineares fracamente acoplados com parâ metros. Tese apresentada ao Departamento de Matemática da Universidade de Brasília.

[17] Ghosh, M. K.; Arapostathis, A.; Marcus, S. I., Optimal Control of Switching Diffusions with Application to Flexible Manufacturing Systems. Siam J. Control and Optimization 31 (1992), 1183-1204.

[18] Györi, I.; Hartung, F.; Mohamady, N. A. Existence and uniqueness of positive solutions of a system of nonlinear algebraic equations. Period. Math. Hung. 75 (2017), no. 1, 114-127.

[19] Györi, I.; Hartung, F.; Mohamady, N. A. Boundedness of positive solutions of a system of nonlinear delay differential equations. Discrete and Continuous Dynamical Systems - Series B. 23 (2018), no. 2, 809-836.

[20] Ishii, H. 'Perron's method for Hamilton-Jacobi equations'. Duke Math. J. 55 (1987), 369-384.

[21] Ishii, H.; Koike, S. Viscosity Solutions of a System of Nonlinear Second-Order Elliptic PDEs Arising in Switching Games. Funkcialaj Ekvacioj 34 (1991), 143-155.

[22] Katsoulakis, M.; Koike, S. Viscosity solutions of monotone systems for Dirichlet problems. Differential Integral Equations 7 (1994), 367-382.

[23] Kawano, N. On bounded entire solutions of semilinear elliptic equations. Hiroshima Math. J. 14 (1984), $125-158$.

[24] Lasry, J. M.; Lions, P. L. Nonlinear Elliptic Equations with Singular Boundary conditions and Stochastic Control with State Constraints. Math. Ann. 283 (1989), 583-630.

[25] Lee E. K.; Shivaji, R.; Ye, J. Classes of infinite semipositone systems. Proc. Roy. Soc. Edinburgh Sect. A. 139A (2009), 853-865.

[26] Mao, X. Stability of stochastic differential equations with Markovian switching. Stochastic Process. Appl. 79 (1999), 45-67.

[27] Nesterov, Y. Introductory lectures on convex optimization: A basic course. Springer, 2004.

[28] Oksendal, B. Stochastic Differential Equations. Springer-Verlag, 5th Ed, 2000.

[29] Song, Q.; Zhu, C., On singular control problems with state constraints and regime-switching: A viscosity solution approach. Automatica 70 (2016), 66-73.

[30] Pirvu, T. A.; Zhang, H. Investment-consumption with regime-switching discount rates. Math. Social Sci. 71 (2014), 142-150.

[31] Tolksdorf, P. On the Dirichlet problem for quasilinear equations in domains with conical boundary points. Commun. PDEs. 8 (1983), 773-817.

[32] Zhou, X. Y.; Yin, G. Markowitz' os mean-variance portfolio selection with regime switching: a continuoustime model. SIAM J. Control Optim 42 (2003), 1466-1482.

[33] Yao, D. D.; Zhang, Q.; Zhou, X. Y. A Regime-Switching Model for European Options. Stochastic Processes, Optimization, and Control Theory Applications in Financial Engineering, Queueing Networks, and Manufacturing.

[34] Zhu, C. On singular control problems with state constraints and regime-switching: A viscosity solution approach. Automatica 70 (2011), 1570-1579.

\author{
THE BuCHAREST UNIVERSITY OF ECONOMIC STUDIES \\ DEPARTMENT OF APPLied MATHEMATICS \\ Piata RomAnA, 1St District, Bucharest, Postal Code: 010374, POSTAL OfFICE: 22, RomANiA \\ Email address: dragos. covei@csie.ase.ro
}

MCMASTER UNIVERSITY

DEPARTMENT OF MATHEMATICS AND STATISTICS

1280 Main Street West, Hamilton, ON, L8S 4K1, Canada

Email address: tpirvu@math.mcmaster.ca 